\title{
Nanoporous carbon superstructures based on covalent bonding of porous fullerenes
}

\section{A R T I C L E I N F O}

Article history:

Received 22 November 2017

Received in revised form

8 January 2018

Accepted 9 January 2018

Available online 12 January 2018

\begin{abstract}
A B S T R A C T
Highly porous and mechanically stable nanostructures are of great interest for applications in selective membranes, adsorbents, catalysts and sensors. In this study, we use Density Functional Theory calculations and Molecular Dynamics (MD) simulations to demonstrate the feasibility of a novel class of porous carbon-based nanostructures with uniform pore size distributions, formed by covalent bonding of porous fullerenes. Their corresponding mechanical and electronic properties are evaluated, and results show that they typically exhibit an outstanding mechanical strength and electronic behavior ranging from metallic to semiconducting, depending on the hybridization of the covalent interconnections and dimensionality. The efficacy of these materials as molecular sieves is also demonstrated using MD simulations of gas transport across the nanoporous structure. This combination of properties makes these nanostructures suitable for the development of novel porous functional materials with several potential applications.
\end{abstract}

(c) 2018 Elsevier Ltd. All rights reserved.

\section{Introduction}

Nanoporous materials are characterized by the presence of pores smaller than $100 \mathrm{~nm}$, exhibiting a large specific surface area, and typically a narrow pore size distribution (PSD) [1]. Examples of materials with such features are activated carbon, metal-organic frameworks, zeolites among others. Due to these set of characteristics, nanoporous materials have been successfully used as gas storage media and catalyst supports, applied in membrane separation processes and in the development of sensors, among other potential applications [1-5]. The pores can also be impregnated with other species (metal nanoparticles, ions, biomolecules) extending the horizon of applications of this family of materials $[3,6]$. Advances in the synthesis of carbon nanomaterials have brought new possibilities of creating carbon-based nanoporous structures with customized pore size distributions and with enhanced physical properties (mechanical, electronic, thermal), enabling their use as multi-functional materials, toward the development of innovative applications [3,7-9]. Mathematical and

\footnotetext{
* Corresponding author.

E-mail address: amuniz@enq.ufrgs.br (A.R. Muniz).
}

theoretical approaches have also been used to propose novel nanoporous 3-D carbon-based materials, with "exotic" atomic configurations, varied porosity and interesting electronic properties [10,11].

One interesting class of such materials is the so called porous fullerenes, introduced by Paupitz et al. [12]. These cage-like carbonbased configurations are conceptually formed by wrapping up either porous graphene [13] (PG) or biphenylene-carbon [14] (BPC) monolayers, analogously to the formation of fullerenes from graphene sheets. A significant variety of stable fullerenic configurations (totaling 18 possibilities) containing 120 up to 540 atoms have been proposed using Density Functional-based Tight-Binding calculations and Molecular Dynamics simulations. These porous fullerenes have been suggested to be suitable for gas storage and molecular encapsulation. Carbon nanomaterials (fullerenes [15-18], carbon nanotubes [19] and graphene [20]) have been successfully used as building blocks and as architectural models for the creation of novel superstructures with different set of properties, such as carbon nanotube networks, graphene-nanotube composites, among many others [7]. There are several studies demonstrating the possibility of polymerization of conventional fullerenes $\left(\mathrm{C}_{60}\right)$ toward the creation of larger structures, achieved by connecting two or more fullerenes through covalent bonds on 
their surface $[17,18]$ or fullerene coalescence under confinement within carbon nanotubes [21]. Such structural transformations can be induced using different physical and/or chemical processes, such as application of high pressures, plasma treatment, and ion or electron irradiation $[17,18,21]$. In principle, similar approaches could be applied to the aforementioned porous fullerenes, generating analogous superstructures.

In the present work, we present a comprehensive computational study employing Density Functional Theory (DFT) calculations and classical Molecular Dynamics (MD) simulations to demonstrate the feasibility of superstructures formed by covalent bonding of porous fullerenes and compute some relevant physical properties. We show that one-, two-, and three-dimensional (1-D, 2-D and 3-D) nanostructures with varied pore size distribution can be generated using this principle. These structures exhibit a high mechanical strength as demonstrated in uniaxial tensile strain tests, and an electronic behavior ranging from metallic to semiconducting, depending on the nature of the fullerene interconnections and dimensionality. We also show that the narrow pore size distribution typical of these materials enable their use as molecular sieves in gas separation processes.

\section{Computational methods}

\subsection{Molecular dynamics simulations}

The atomic structure of the porous fullerene-based superstructures and their corresponding mechanical and gas permeation properties were computed by classical molecular dynamics simulations using the package LAMMPS [22]. For structural optimization and computation of mechanical properties, the interatomic interactions were described using either the Adaptive Interatomic Reactive Bond-order potential (AIREBO [23]) or the Reactive Force Field (ReaxFF [24]), both widely used in the prediction of structural and mechanical properties of carbon based nanomaterials. Two distinct parameterizations were used for ReaxFF, labeled herein as ReaxFF-FC and ReaxFF-RevC. The first was fitted to study C/Ni chemistry and extended to functionalized graphene [25], and the second was developed to describe the chemistry of condensed carbon phases and revised to study defects in graphite and include $\mathrm{C}-\mathrm{H}$ bonds [26]. For the simulations using AIREBO, we set the $\mathrm{C}-\mathrm{C}$ cutoff distance to $2.0 \AA$ to avoid spurious high forces at higher strains, as done in previous works $[27,28]$. For the analysis of gas transport through the nanostructures, the Lennard-Jones (LJ) potential in conjunction with the coulombic interaction potential were used to describe the interactions between gas molecules and the solid surfaces. The LJ parameters and atomic charges for the molecules $\left(\mathrm{H}_{2}, \mathrm{O}_{2}, \mathrm{~N}_{2}, \mathrm{CO}, \mathrm{CH}_{4}\right)$ and solid carbon were extracted from Refs. [29-31]; these parameters are derived from the OPLS force field and have been previously used to study gas transport across graphene-based membranes and gas adsorption on graphitic surfaces [29,31]. Also, the atoms of the porous carbon matrix were kept static in these simulations (only gas molecules were mobile), and the molecular bond lengths were kept constant during the simulation using the SHAKE algorithm, as done in previous similar works [31].

Structural optimization was achieved by carrying out simulations at constant temperature $(0.1 \mathrm{~K})$ and zero stress, employing the Berendsen thermostat and barostat [32]. Periodic boundary conditions are applied in all directions, using supercell sizes defined according to a preliminary convergence study. For the evaluation of mechanical properties upon uniaxial tensile strain, the structures are continuously strained (rate of $0.005 \mathrm{ps}^{-1}$ ) at a constant temperature $(300 \mathrm{~K})$ until fracture is observed. Zero-stress condition in the direction perpendicular to the applied strain is ensured by using the Berendsen barostat. The corresponding stress-strain (SS) curves are obtained by computing the atomic virial stress along the trajectories. The atomic volumes of the superstructures required on the stress calculation were computed using the volume of a single fullerene $V_{F}$ as basis (for example, a 2-D supercell containing $\mathrm{N}$ porous fullerenes have an atomic volume of $N V_{F}$ ). The Young modulus, tensile strength and fracture strain are directly extracted from these curves, and the Poisson ratio is computed from measured dimensional changes in the simulation box.

The assessment of gas transport and separation properties of selected nanoporous fullerene-based configurations were also carried out using MD simulations. More specifically, we created a simulation box divided in two equal parts, delimited by the nanoporous structure (acting as a membrane module). One half of the box is initially filled with the molecules, while the other is empty. Simulations are then carried at constant temperature of $300 \mathrm{~K}$ (controlled by the Nose-Hoover thermostat), and we count the numbers of molecules of a given species $i$ on each side of the system as function of time $N_{1, i}(t)$ and $N_{2, i}(t)$ (labels 1 and 2 correspond to the initially filled and emptied compartments, respectively). The slope of the curve $N_{2, i}(t)$ at initial times $W_{i}=d N_{2, i} / d t$ provides an estimate for the initial rate of mass transfer through the material (when the imposed concentration gradient is a maximum). The ideal membrane selectivity for a pair of gases A and B is defined as the ratio between the corresponding rate of mass transfer measured for the pure species, $S_{A / B}=W_{A} / W_{B}$; this parameter has been traditionally used in both experimental and computational studies to evaluate the separation efficiency of membranes from data obtained in pure gas permeation tests [33-35]. This selectivity provides a simple and reliable metric for the efficiency of the membrane to separate a species A from a gaseous mixture containing both $\mathrm{A}$ and $\mathrm{B}$ species [33,35].

\subsection{Density Functional Theory calculations}

The atomic and electronic structure of selected fullerene-based nanoporous configurations were computed using DFT calculations as implemented in the QUANTUM ESPRESSO package [36]. These calculations were carried out within the generalized gradient approximation (GGA/PBE), employing plane waves for the expansion of Kohn-Sham orbitals, with an energy cutoff of 45 Ry, and PAW pseudopotentials for treating valence and core electrons. Considering the large number of atoms in the supercell (up to 216 atoms), only the gamma point was used for sampling the first Brillouin zone in the electron density calculations. A vacuum of at least $15 \AA$ was used to avoid spurious interactions between images in 1-D and 2-D configurations. Marzari-Vanderbilt cold smearing was applied with a smearing factor of $0.020 \mathrm{Ry}$. Atomic structures were relaxed with respect to their internal atomic positions and supercell lengths within a force tolerance of $10^{-4}$ Ry/bohr. Electronic band dispersions were computed along conventional paths in the 1 st Brillouin zone, including the special symmetry points.

\section{Results and discussion}

\subsection{Atomic structure}

A broad family of porous fullerenes originated from BPC and PG monolayers has been introduced by Paupitz and coworkers [12], with diameters ranging from 10 to $22 \AA$. Despite the rich variety of configurations, we chose the $\mathrm{C} 24$ porous fullerene $\left(\mathrm{C}_{144} \mathrm{H}_{72}\right)$ as the building block for our superstructures in this work. It contains structural features which are representative of the remaining members of family [12]. The atomic structure of this fullerene is illustrated in Fig. 1(a), conceptually generated from porous 
(a)

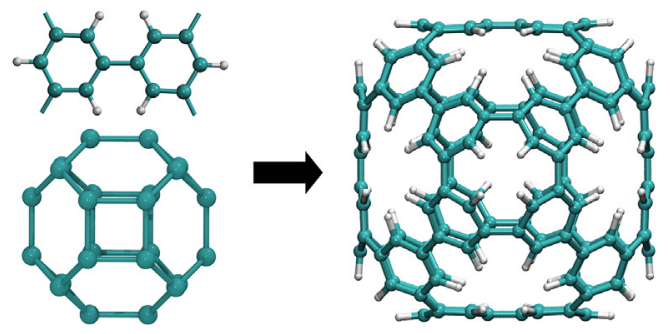

1-dimensional

(b)

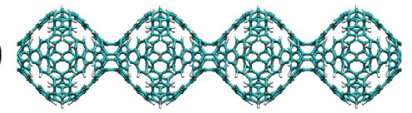

(c)

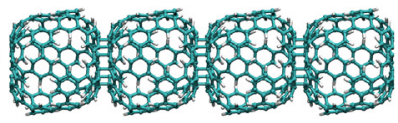

(d)

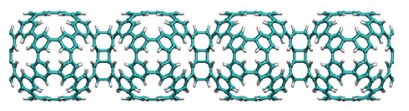

2-dimensional

(e)

(f)
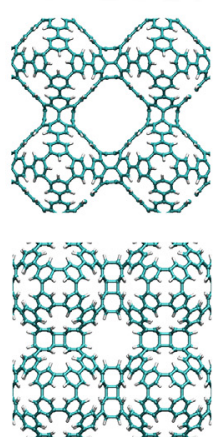

3-dimensional

(g)

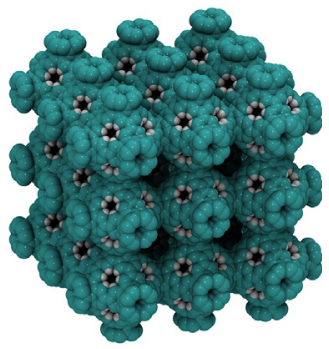

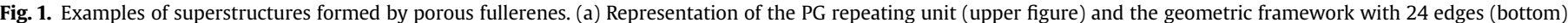

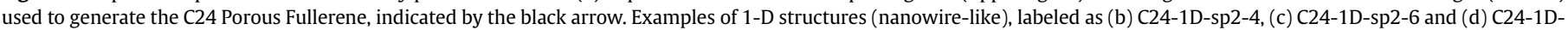
sp3-4, 2-D structures, labeled as (e) C24-2D-sp2-4 and (f) C24-2D-sp3-4, and a bulk 3-D (g) C24-3D-sp2-4 configuration.

graphene monolayers in a total of 24 units arranged in the depicted caged framework.

The connections between the porous fullerenes (PF) toward creation of larger superstructures were established using two approaches. One of them consists in connecting directly the fullerenes through covalent bonding of the functionalized $C$ atoms near the pores ( $s p^{2}$-hybridized), resulting in $s p^{3} \mathrm{C}-\mathrm{C}$ bonds between equivalent atoms from two neighboring PFs, as depicted in Fig. 1(d). The other approach consists in removing the passivating $\mathrm{H}$ atoms from the $\mathrm{C}$ atoms at the pores, and establishing a $\mathrm{C}-\mathrm{C}$ bond connecting the equivalent atoms with dangling bonds of two neighboring fullerenes, as illustrated in Fig. 1(b and c). These carbon atoms are also three-fold coordinated, and the established bonds can be characterized as $s p^{2}$-hybridized, with some slight deviation from the conventional planar $s p^{2}$-hybridized C-C bonds (analogously to $\mathrm{C}-\mathrm{C}$ bonds on the curved surfaces of carbon nanotubes). These connections were chosen based on a preliminary study, taking into account all the possible geometric alignments between pores for a pair of PFs, and checking which ones are the most favorable from a geometric and energetic point of view. In the present study, we did not attempt to investigate possible mechanisms and associated energy barriers involved in the creation of such bonds. They are expected to be induced by specific processes (e.g, high pressure or shock treatment, ion/electron irradiation), as observed experimentally for fullerene polymers $[17,18]$ and coalesced fullerenes within carbon nanotubes [21].

These connections were used to create a series of one-, two- and three-dimensional superstructures based on C24 porous fullerenes as building blocks. Some examples are depicted in Fig. 1(b-g). More examples are shown in the SI. The 1-D superstructures (Fig. 1(b-d)) consist in flexible chains of porous fullerenes, which resemble the "fullerene polymers" created by direct covalent bonding of conventional $C_{60}$ fullerenes [17] and the 1-D tubular structures achieved by coalescence of fullerenes under confinement [21]. The 2Dand 3D-configurations can be seen as an extension of these interconnections along two and three directions, so every fullerene is bonded to either 4 or 6 neighbors, forming a planar square or a simple cubic lattice respectively, as seen in Fig. 1(e-g). More complex 2D and 3D crystalline arrangements could be, in principle, generated limited by the symmetry of the fullerene and possible alignment of pores. These materials are essentially nanoporous, and visual inspection shows that pores of varying diameters are present: two pores are intrinsic of the surface of the porous fullerene ( 2.5 and $3.9 \AA$, same as from original porous graphene), one corresponding to the interior of the fullerenes $(9 \AA)$, and others (e.g., $9.7 \AA$ in Fig. 1(e) and $6.7 \AA$ in Fig. 1(f)) arise due to the spatial arrangement of the basic blocks. If we manipulate the number of neighboring fullerenes and/or the symmetry of the lattice, as well the size/type of porous fullerene, we can generate structures with varied pore size distributions and characteristic dimensions, as shown in the SI. Due to the presence of these pores, these materials are expected to have a lower density compared to solid carbon allotropes; for example, the bulk 3-D configuration shown in Fig. $1(\mathrm{~g})$ have a density of $0.821 \mathrm{~g} / \mathrm{cm}^{3}$, while diamond and graphite exhibit densities around 3.5 and $2.1 \mathrm{~g} / \mathrm{cm}^{3}$ respectively.

The structures created were labeled based on the PF used, their dimensionality, the type of inter-fullerene connection, and the number of covalent bonds used to establish the connection. For example, the 1-D configurations depicted in Fig. 1(b) and (c) were created by C24 fullerenes connected through respectively four and six $s p^{2}$ bonds on each pore, therefore denominated "C24-1D-sp2-4" "C24-1D-sp2-6"; the configuration shown in Fig. 1(d) was created by connecting the same fullerene through four $s p^{3}$ bonds on each pore, hence labeled as "C24-1D-sp3-4".

The structures were optimized according each method described in Sec. 2, i.e. using DFT calculations and MD simulations with interatomic interactions described either by AIREBO or ReaxFF. DFT and ReaxFF were able to predict stable structures containing all types of inter-fullerene connections as depicted in Fig. 1. AIREBO was not able to predict properly the ones 
characterized by inter-fullerene bond angles significantly lower than $109^{\circ}$, which are present in the structures illustrated in Fig. 1(d,f), for example. In this case, structural relaxation spontaneously led to the distortion in the angles and even a rupture of some bonds, leading to a reduction in the number of inter-fullerene bonds (from 6 to 2). Properties were not computed using AIREBO in these cases.

The relative stability of the configurations were evaluated using a binding energy defined as $E_{b}=\left(E_{\text {sup }}+N_{\mathrm{H}_{2}} E_{\mathrm{H}_{2}}-E_{C 24}\right) / N_{I B}$, where $E_{\text {sup }}, E_{\mathrm{C} 24}$ and $E_{\mathrm{H}_{2}}$ are the energies of the PF-based superstructure, the pristine isolated $\mathrm{C} 24 \mathrm{PF}$ and the $\mathrm{H}_{2}$ molecule respectively. $N_{I B}$ is the number of inter-fullerene covalent bonds created, and $N_{\mathrm{H}_{2}}$ is half of the number of hydrogens atoms removed from the pores to create the $s p^{2}$ bonds. By definition, $N_{H 2}=0$ for the configurations created upon $s p^{3}$ inter-fullerene connections (without removing $\mathrm{H}$ atoms). The binding energies computed for selected configurations using DFT are given in Table 1 . The results show that these energies are slightly positive and within $0.5-1.1 \mathrm{eV}$ per inter-fullerene bond, suggesting that these superstructures are metastable with respect to the isolated fullerenes. As discussed before, we did not attempt to evaluate the energy barriers for creation/dissociation of these bonds.

Additionally, we employed MD simulations at finite temperatures to assess the stability of the 1-D superstructures. Relaxed configurations initially at $0 \mathrm{~K}$ were subjected to a constant heating rate, and the integrity of the inter-fullerene bonds was monitored as function of the temperature. We verified that the structures with $s p^{2}$ and $s p^{3}$ interconnecting bonds remain intact at temperatures as high as 2000 and $1000 \mathrm{~K}$ respectively according ReaxFF, confirming their thermal stability. At these temperatures, dehydrogenation and bond breaking starts to occur. These temperatures exceed those observed experimentally for dehydrogenation of functionalized graphene, for example [37]. The strength of these interfullerene $\mathrm{C}-\mathrm{C}$ covalent bonds was also assessed upon the evaluation of mechanical properties under tensile strain as discussed in Sec. 3.3.

\subsection{Electronic structure}

Carbon nanomaterials exhibit a diverse behavior regarding their eletronic properties, which make them suitable to the development of innovative technologies in nanoelectronics and nanosensors. Metallic, semiconducting and insulating behavior and varied carrier mobilities have been observed for these materials, depending on the hybridization of $\mathrm{C}-\mathrm{C}$ bonds and structural features, enabling several potential applications [38]. Therefore, the electronic structure of the proposed nanostructures deserves being investigated.

Toward this end, we computed the electronic band structure and the integrated local density of states (ILDOS) for a representative set of configurations. The calculations indicate that two factors define the main features of the band structure and of the spatial extension of frontier orbitals (directly related to the electronic behavior of these materials), namely the type of connection

Table 1

Binding energy $\left(E_{b}\right)$ (eV/interlayer bond) for selected structures as predicted by DFT.

\begin{tabular}{ll}
\hline Configuration & $\mathrm{E}_{b}$ \\
\hline C24-1D-sp2-4 & 1.04 \\
C24-2D-sp2-4 & 1.08 \\
C24-3D-sp2-4 & 1.08 \\
C24-1D-sp2-6 & 0.73 \\
C24-1D-sp3-4 & 0.55 \\
C24-2D-sp3-4 & 0.51 \\
\hline
\end{tabular}

between neighboring fullerenes $\left(s p^{2}\right.$ or $s p^{3}$ hybridized C-C bonds, as defined in Sec. 3.1) and the dimensionality of the superstructure (1-D, 2-D and 3-D).

Electronic band structure plots for some selected configurations are shown in Fig. 2. The 1-D configurations exhibited semiconducting behavior; the computed electronic band gaps as predicted by DFT/GGA were of $1.73,0.75$ and $0.35 \mathrm{eV}$ for the C24-1Dsp3-4, C24-1D-sp2-4 and the C24-1D-sp2-6 configurations, respectively. Similar behavior was found for the 2-D nanostructures, with gaps of 1.52 and $0.05 \mathrm{eV}$ for C24-2D-sp3-4 and C24-2D-sp2-4 configurations. For both 1-D and 2-D superstructures, the presence of $s p^{2} \mathrm{C}-\mathrm{C}$ interconnections clearly leads to the smallest band gaps. Also, the band gaps are decreased for a given type of interconnecting bonds when 1-D structures are analogously extended to 2-D and 3-D. Interestingly, the bulk 3-D configuration C24-3D-sp2-4 exhibited a metallic behavior, as seen in the band structure of Fig. 2(c). It is worth mentioning that the 3-D nanoporous graphene-based materials theoretically introduced in Ref. [11] also exhibit a varied electronic behavior (from metallic to semiconducting) depending on details of their atomic structure. The 1-D tubules formed by coalescence of fullerenes within nanotubes reported in Ref. [21] were also found to be either metallic or semiconducting (the latter with a band gap of $1.65 \mathrm{eV}$, close to the values observed in the present study). These results suggest the possibility of creating carbon-based nanoporous materials with tunable electronic properties, which can explored toward the
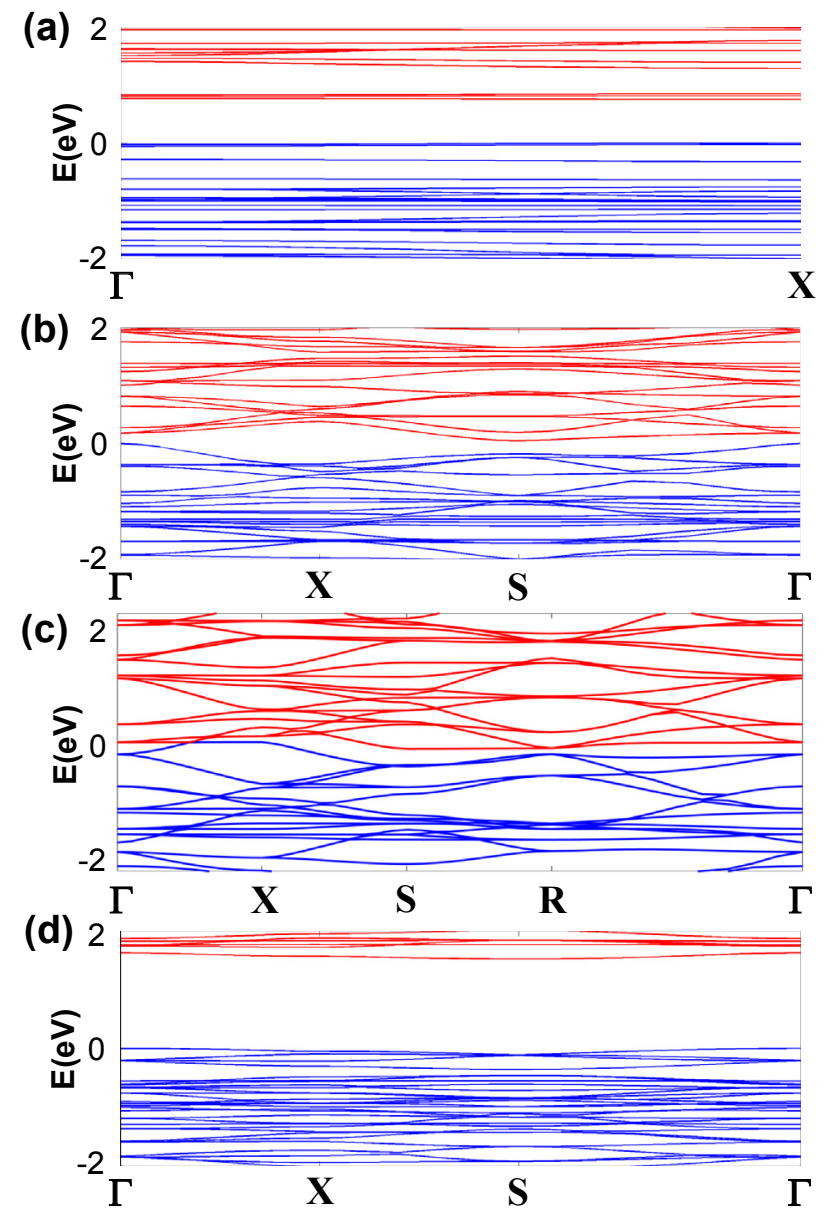

Fig. 2. Electronic band structures in the vicinity of Fermi level $(E=0)$ for $(a) C 24-1 D-$ sp2-4, (b)C24-2D-sp2-4, (c)C24-3D-sp2-4 and (d)C24-2D-sp3-4 configurations. Blue and red curves (color online) correspond to the valence and conduction bands respectively. 
development of several applications in sensors and nanoelectronics $[1,8]$.

Isosurfaces and contour plots of the ILDOS, integrated over energy ranges around the top of valence band and the bottom of conduction band, help to understand the differences in the electronic band structures of the proposed configurations with $s p^{2} \mathrm{C}-\mathrm{C}$ interconnections. These plots are depicted in Fig. 3. The ILDOS plots for the C24-1D-sp2-4 configuration depicted in Fig. 3(a,d,g) show that the frontier orbitals $\left(p_{z}\right)$ are quite localized around the fullerene $s p^{2}$-hybridized $\mathrm{C}-\mathrm{C}$ interconnections. There is no delocalization of these orbitals along the main axis, suggesting that the electronic transport may not be favoured in the material. This observation is also confirmed by analyzing the corresponding band structure depicted in Fig. 2(a), characterized by the presence of flat bands around the Fermi level, indicating large effective masses and consequently, low carrier mobilities.

A similar analysis was carried out for the C24-2D-sp2-4 configuration (based on the same type of interconnection), and the corresponding ILDOS plots are shown in Fig. 3(b, e and h). In this case, a larger degree of electron delocalization along the two orthogonal directions of the plane is observed, which would favor the electronic carrier mobility of the material. The band structure depicted in Fig. 2(b) reflects this observation; the gap is significantly reduced, and the energy dispersion around the Fermi level is parabolic-like, indicating that carriers effective masses should be smaller than those of the analogous 1-D materials, potentially enhancing the electronic transport. In the case of the 3-D counterpart (C24-3D-sp2-4), the ILDOS contour plot (Fig. 3(c, f and i)) shows a significant electron delocalization along all directions, suggesting that the carriers could be nearly free to move throughout the structure. This observation is corroborated by its corresponding band structure (Fig. 2(c)), characteristic of a metallic material.

\subsection{Mechanical properties}

Carbon-based nanostructures (graphene, carbon nanotubes, diamond nanothreads, carbyne chains) are well-known to exhibit outstanding mechanical properties, exceeding those of conventional engineering materials [7,27,28,38]. Therefore, it would be reasonable to expect a similar behavior for the PF-based superstructures introduced in the present study. MD simulations of uniaxial tensile strain tests were used to evaluate the mechanical strength of the proposed PF-based configurations as described in Sec. 2.1.

Examples of stress-strain curves obtained in these tests are depicted in Fig. 4, and the values for the measured properties are summarized in Table 2. Results are shown as predicted by both AIREBO, ReaxFF-revC and ReaxFF-FC; deviations among the predictions with these different interatomic potentials are expected, as demonstrated in previous studies [28,39]. Despite these observed deviations, these potentials have been demostrated to perform

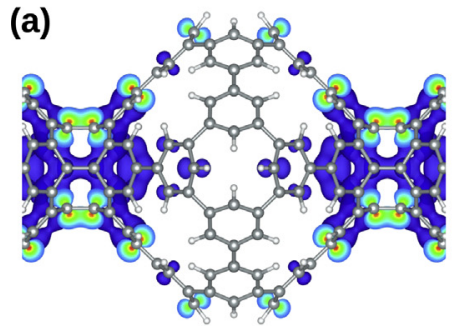

(d)

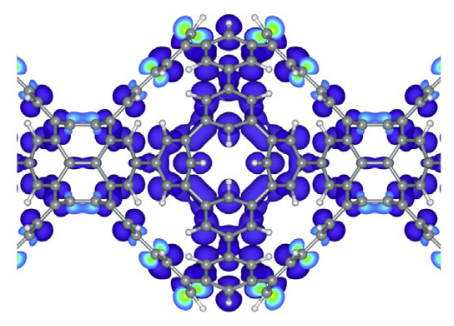

(g)

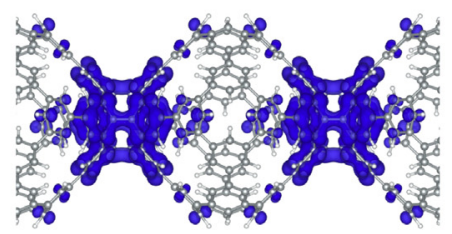

(b)

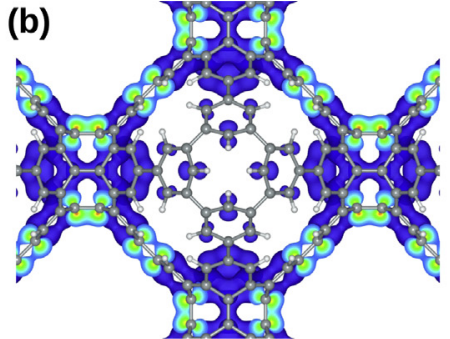

(e)

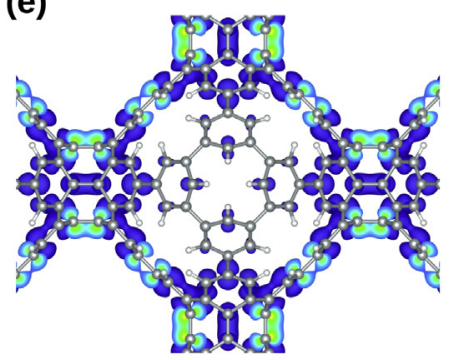

(h)

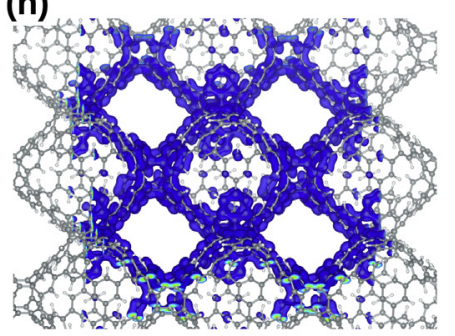

(c)

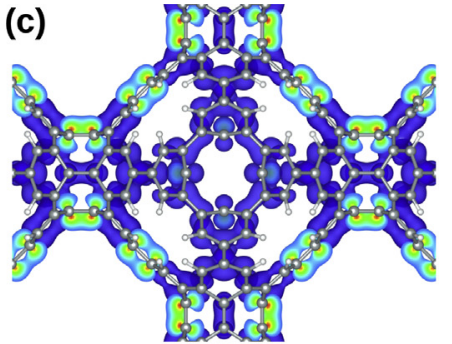

(f)
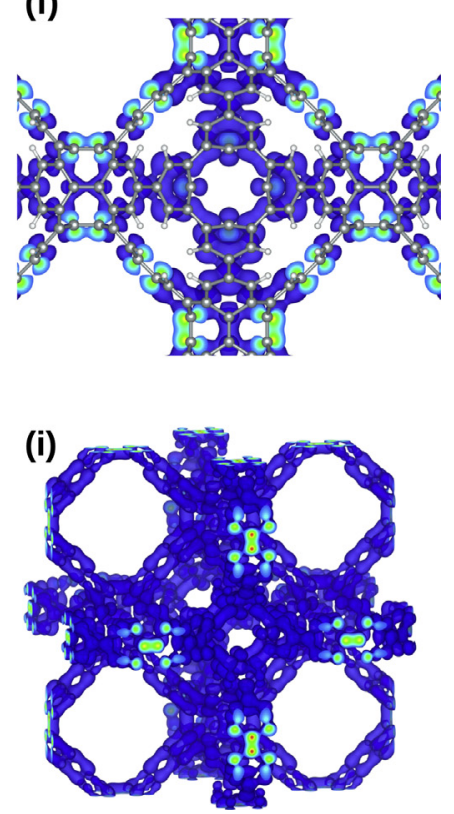

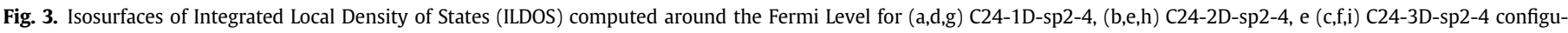

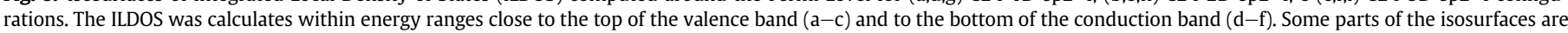

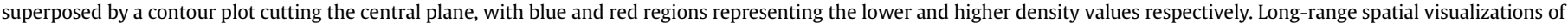

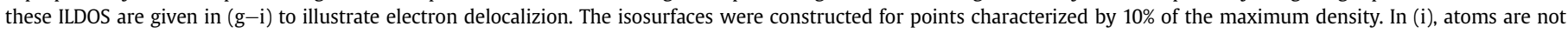
shown to allow a clearer visualization of the continuous 3-D fully connected network formed by ILDOS in that case. 

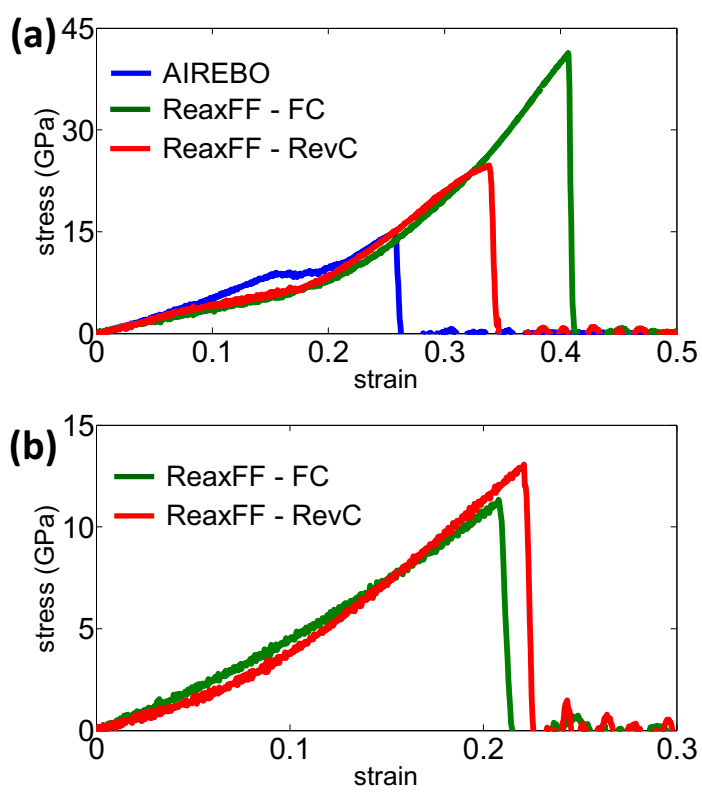

Fig. 4. Stress-strain curves obtained in MD tensile strain tests for (a)C24-1D-sp2-4 and (b)C24-1D-sp3-4 configurations, as predicted by different interatomic potentials and parameterizations.

Table 2

Mechanical properties of the porous fullerene-based superstructures and conventional carbon nanostructures computed by different interatomic potentials.

\begin{tabular}{|c|c|c|c|}
\hline \multirow[t]{2}{*}{ Configuration } & $\mathrm{T}(\mathrm{GPa})$ & $\varepsilon$ & $\mathrm{Y}(\mathrm{GPa})$ \\
\hline & AIREBO $/ \mathrm{R}_{F C} / \mathrm{R}_{\text {Rev } C}$ & AIREBO $/ \mathrm{R}_{F C} / \mathrm{R}_{\operatorname{RevC}}$ & AIREBO/R $\mathrm{R}_{F C} / \mathrm{R}_{\operatorname{RevC}}$ \\
\hline C24-1D-sp2-4 & $15 / 41 / 25$ & $0.26 / 0.41 / 0.34$ & $44 / 39 / 40$ \\
\hline C24-1D-sp2-6 & $22 / 56 / 34$ & $0.20 / 0.33 / 0.26$ & $71 / 62 / 68$ \\
\hline C24-1D-sp3-4 & $-/ 11 / 13$ & $-/ 0.21 / 0.22$ & $-/ 38 / 28$ \\
\hline C24-2D-sp2-4 & $14 / 48 / 21$ & $0.28 / 0.40 / 0.31$ & $27 / 33 / 32$ \\
\hline C24-2D-sp3-4 & $-/ 7 / 10$ & $-/ 0.17 / 0.21$ & $-/ 32 / 19$ \\
\hline CNT-(6,6) & $102 / 128 / 128$ & $0.21 / 0.14 / 0.22$ & $841 / 823 / 836$ \\
\hline SLG-ZZ & $113 / 140 / 144$ & $0.21 / 0.17 / 0.27$ & $845 / 752 / 717$ \\
\hline SLG-AC & $91 / 103 / 143$ & $0.14 / 0.12 / 0.26$ & $933 / 738 / 706$ \\
\hline
\end{tabular}

${ }^{*} \mathrm{~T}$ is the ultimate strength, $\varepsilon$ is the fracture strain and $\mathrm{Y}$ is the Young modulus of the nanostructures as computed by AIREBO, ReaxFF-FC $\left(\mathrm{R}_{F C}\right)$ and ReaxFF-RevC $\left(\mathrm{R}_{\operatorname{RevC}}\right)$ respectively for each entry. SLG-ZZ and SLG-AC indicate graphene layers in zigzag and armchair configurations respectively, while CNT- $(\mathrm{n}, \mathrm{m})$ indicate carbon nanotubes with chiralities represented by the indices $n$ and $m$. The "-" values were not computed due to inability of the corresponding potential to properly describe a specific nanostructure, as discussed in the text.

quite well when compared to experimental or DFT calculated data for carbon nanostructures. We employed these potentials to compute the properties for a representative armchair CNT (with a diameter similar to those of the fullerene with $s p^{2}$ interconnections) and single layer graphene (also shown in Table 2); these values agree with previous studies $[27,39,40]$ and show the aforementioned discrepancies in the predictions. These values are also used as reference states for the properties of our nanostructures. We used these three approaches because of their success in previous similar studies, and considering the possible discrepancies among their predictions, we aimed to establish a reasonable range of expected values for the considered properties.

In general, these materials exhibit a brittle behavior with stiffness (Young modulus) in the range of $20-70 \mathrm{GPa}$ and a tensile strength within 10-56 GPa. Stiffness values are inferior to those exhibited by carbon nanotubes and graphene sheets as predicted by the same potentials (also available in Table 2), and are in the same order of magnitude to those found for engineering materials like aluminum or magnesium. The predicted tensile strengths, despite being smaller than those found for CNTs and graphene, are significantly superior than those exhibited by conventional materials, posing the proposed porous structures as quite strong materials.

It is worth noticing to note that some of the PF-based superstructures have a relatively high fracture strain (around 0.30-0.40), making them significantly flexible when compared to the conventional carbon nanostructures $(0.12-0.27$ for graphene/CNT according predictions from Table 2). Our results indicate that the PFbased configurations characterized by $s p^{3}$ fullerene interconnections are slightly weaker and softer than those created through $s p^{2} \mathrm{C}-\mathrm{C}$ ones. In the case of 2-dimensional structures, the Poisson ratio calculated using ReaxFF for C24-2D-sp2-4, resulted in 0.456 and 0.475 for $\mathrm{R}_{F} C$ and $\mathrm{R}_{\operatorname{Rev} C}$ respectivelly, while for the sp3 connected superstructure identified as C24-2D-sp3-4, we found 0.189 and 0.163 using the same ReaxFF parameterizations. For the 3-dimensional superstructure, our calculations include also the AIREBO potential, and the values found were 0.414, for AIREBO, 0.427 and 0.435 for $\mathrm{R}_{F} C$ and $\mathrm{R}_{\operatorname{RevC}}$ respectively.

An analysis of the evolution of the atomic structure and the corresponding atomic stress distribution during the tensile strain, as shown in Fig. 5, help to understand the mechanical behavior of the material and the reasons for the differences discussed above.

The analysis of these sequences of snaphots show that the stress is evenly distributed throughout the structure in the case of the nanostructure formed by $s p^{2}$ fullerene interconnections (Fig. 5(a,c)); however, stress is concentrated in the $s p^{3} \mathrm{C}-\mathrm{C}$ interconnections in the other case (Fig. 5(b,d)). On both cases, fracture starts at the interconnections, which are characterized as the weakest points of the material. In the first case, due to the even stress distribution, the fullerenes are significantly deformed (flattened, more specifically) before onset of fracture, leading to the high fracture strains reported in Table 2 (up to 0.40). In the second case, stress is concentrated in the interconnections and the fullerenes themselves do not deform significantly before fracture. Therefore, the presence of $s p^{2}$ interconnections makes the material stronger and more flexible. Interestingly, due to the strain applied, fullerene deforms along the direction of applied strain, locally assuming a conformation similar to that of a nanotube in the 1-D case. As one can see in Table 2, these tube-like conformations are weaker when compared to usual nanotubes. This can be attributed to the presence of the $s p^{3}$ interconnections and of vacancies, which would be considered as defects in a usual $s p^{2}$ tubular structure.

\subsection{Gas transport properties}

As discussed in the introduction, nanoporous materials have several practical applications including gas separation, mainly due to the presence of pores with diameters close to the kinetic diameter of molecules of interest. Typical molecular sieves, such as inorganic carbon membranes and zeolites, exhibit narrow PSDs with characteristic diameters up to $10 \AA[1,5]$. The PF-based superstructures introduced in this work have well-defined PSDs, with pore diameters in the range of $2-10 \AA$ (as seen in Sec. 3.1 and depicted in Fig. 6(c)), which enable their use as molecular sieves. There is a growing interest in the development of nanoporous membranes from 2-D materials, such as graphene; their quite small thickness and low density, associated with remarkable mechanical strength and pore size tunability, enable the creation of highly selective membranes and more compact separation modules, which also leads to a reduction in the energy consumption required by the separation process $[9,41-43]$. The PF-superstructures also meet these requirements as demonstrated in the previous sections, and their use as selective membranes for gas separation deserves be evaluated. 
(a)

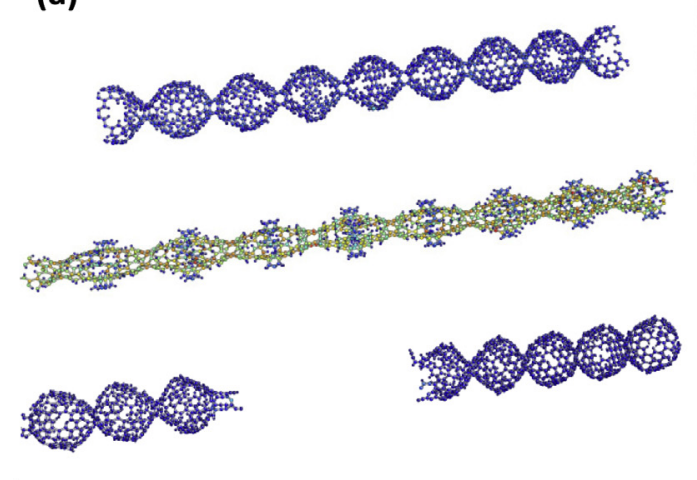

(b)

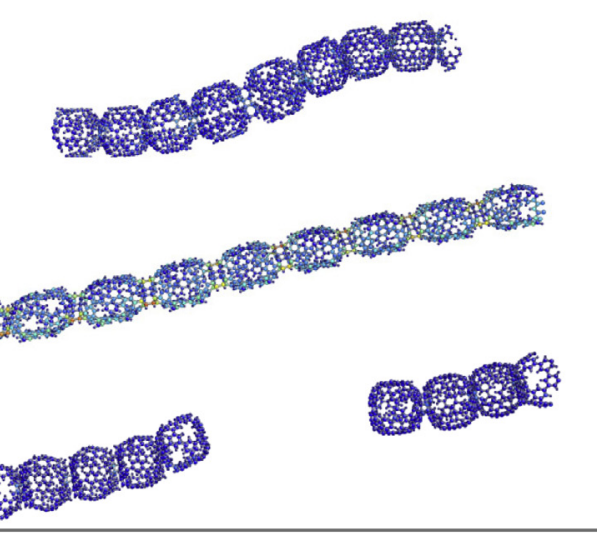

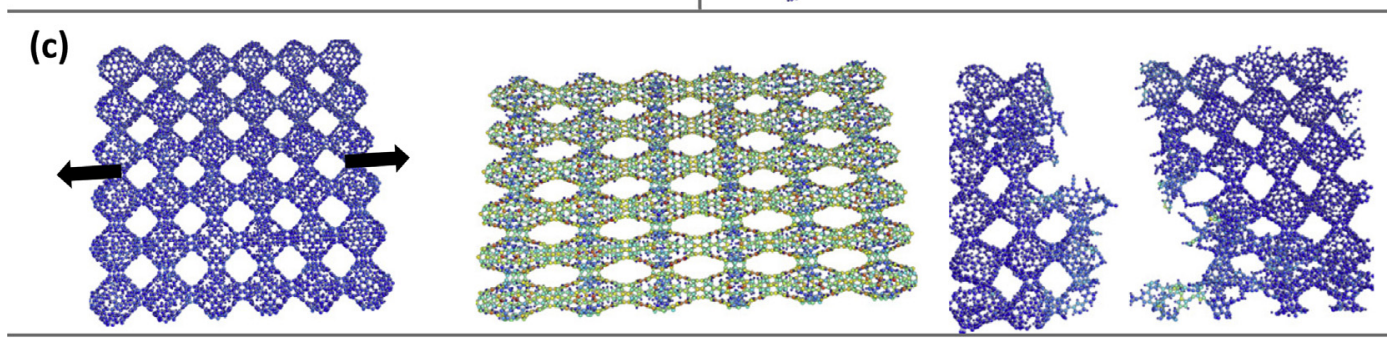
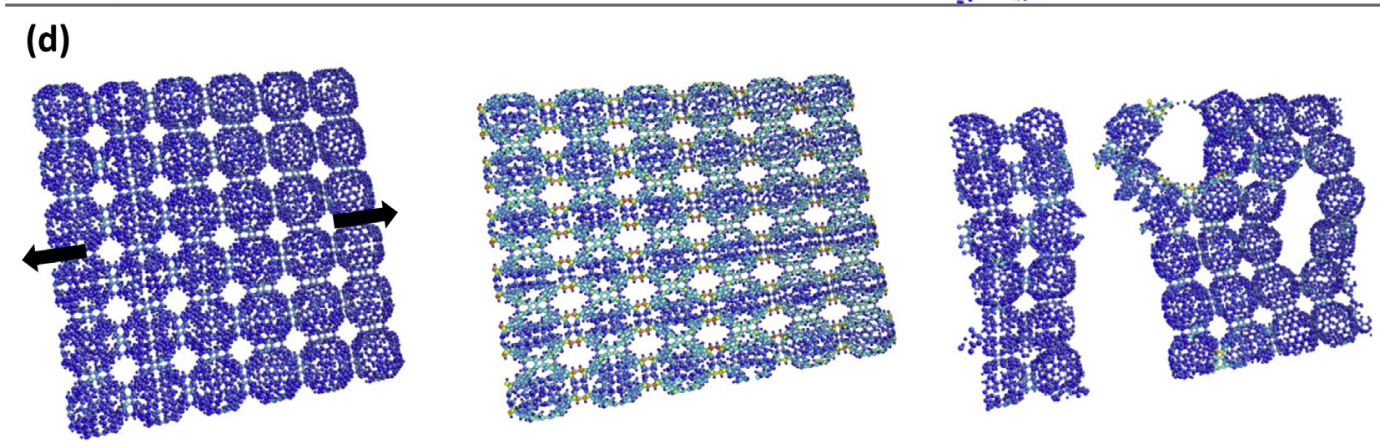

0

atomic stress $\times$ volume $\left(\mathrm{GPa} \cdot \AA^{3}\right) \quad 1500$

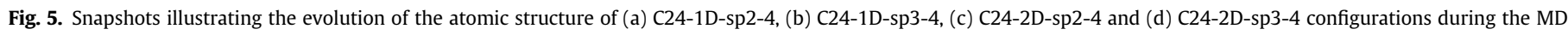

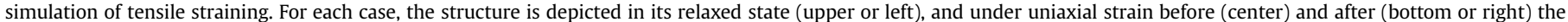
onset of fracture. Arrows depict the direction of application of strain. Atoms are colored according to the atomic von Mises stress (scale shown at the bottom).

As discussed in Sec. 2.1, we carried out MD simulations to evaluate the transport of selected gases within some PF-based superstructures. More specifically, we used the previously relaxed C24-2D-sp3-4 configuration to create "membrane modules", by stacking two (as depicted in Fig. 6(a)), three and four planes. The relative alignment between layers and the equilibrium interplanar distance (defined by the van de Waals interactions) were previously computed using MD simulations. The corresponding PSD of the resulting membrane is shown in Fig. 6(c). The stacking of the 2-D layers generate a new pore size distribution for the material, with characteristic diameters $\left(D_{1}\right.$ - inside the PFs; $D_{2}$ and $D_{3}$ - space within planes) that can be finely controlled by the interplanar spacing as discussed later. The density of the membrane is also quite low $\left(0.896 \mathrm{~g} / \mathrm{cm}^{3}\right)$, analogously to the nanoporous bulk configuration discussed in Sec. 3.1.

We used each membrane to divide the simulation box in two equal parts (one empty and other filled with molecules) as described in Sec. 2.1, and illustrated in Fig. 6(a). We then carried out simulations individually for each gas and each membrane according to the methodology described in Sec. 2.1, resulting in a net mass transfer toward the empty box until equilibrium was reached. Fig. 6(b) depicts an instantaneous snapshot of the process. At least five independent simulations (starting from different initial conditions with respect to atomic position and velocities) were carried out for each situation to ensure statistical significance, always starting with the same number of molecules on side 1 (1000 molecules).

Examples of curves for the number of molecules on the initially empty side (2) as function of time $N_{2, i}(t)$ is given in Fig. 6(c). The initial rate of mass transfer $W_{i}$ is then obtained from linear regression of the curves at initial times. The measured rates (averaged over the independent runs) for each species and for the membranes containing different number of layers are used to compute the initial selectivities as described in Sec. 2.1. These computed rates show that the mass transfer rate for the gases follows the expected order of molecule kinetic diameter $\left(\mathrm{CH}_{4}>\mathrm{CO}\right.$ $>\mathrm{N}_{2}>\mathrm{O}_{2}>\mathrm{H}_{2}$ ) [34,35]; the smaller the diameter, the higher the rate. This observation confirms that the PF-based superstructures can be used as molecular sieves as expected, being able to separate gaseous mixtures based on the difference of molecular sizes. Some selected ideal selectivities $S_{A / B}$ are given in Fig. 6(e), for several pairs of gases of practical interest (e.g. $\mathrm{H}_{2} / \mathrm{CH}_{4}$ for hydrogen removal from natural gas, $\mathrm{CO} / \mathrm{H}_{2}$ for syngas composition adjustment, $\mathrm{O}_{2} / \mathrm{N}_{2}$ for air separation, among others [34]). These values are in the same 

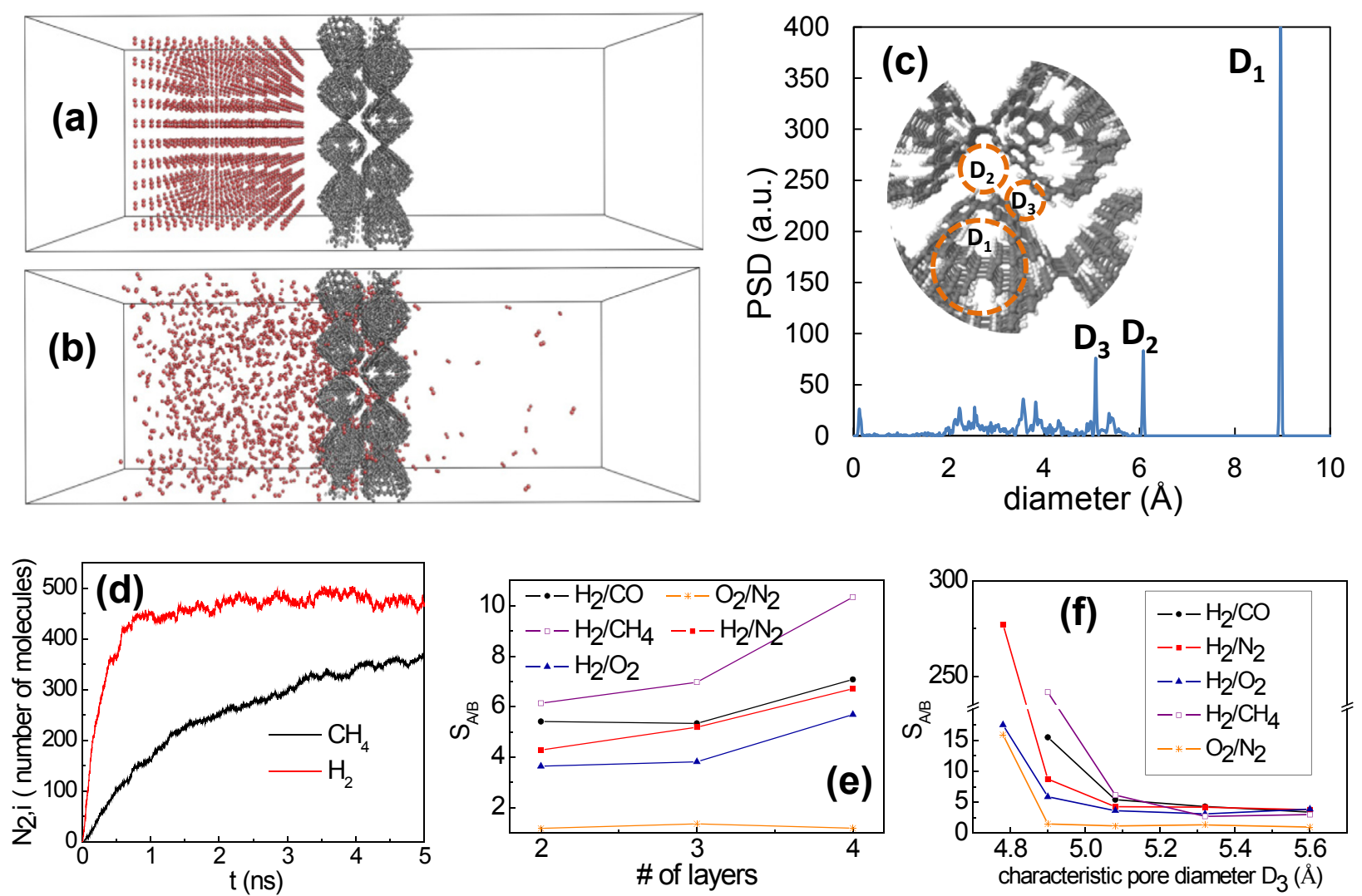

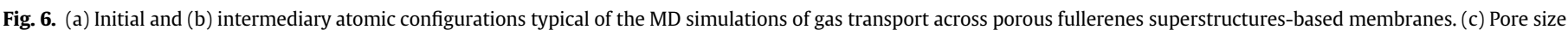

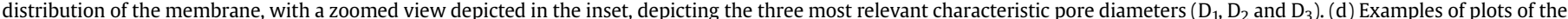

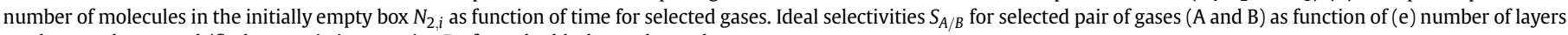
on the membrane and (f) characteristic pore size $\mathrm{D}_{3}$ for a double-layered membrane.

order of magnitude of those exhibited by materials used in conventional membranes for gases separation, such as polymeric membranes, carbon membranes, and zeolites [5,33-35], however not as high as the ones with the highest performances. The increase on the number of layers of the membrane leads to a decrease on the absolute mass transfer rate as expected from mass transfer theory ( $W \propto 1 /$ thickness), however the selectivities are not significantly affected.

We finally investigated how the compactation of the stacked planes in the membrane affects the ideal selectivities. The PSDs of the membranes can be altered by changing the distance between the planes with respect to the equilibrium distance, due to the variation in the diameter of pores associated to the interplanar region (as seen in Fig. 6(c)). Simulations were carried for membranes with slightly larger and smaller interplanar spacings compared to the equilibrium value, and the results are summarized in Fig. 6(f). While the increase on the spacing does not affect substantially the selectivities, minor variations toward a compactation of planes can lead to a significant increase in the selectivities due to smaller pore diameters. This result suggests that a finer control of the compactation of planes (through application of pressure or structural/surface modification), may lead to membranes with higher selectivities, which would be closer and even higher than those of the already existing materials [5,33-35].

The good performance of these materials as molecular sieves, associated with their remarkable mechanical strength, low density and high thermal stability (as demonstrated in Sec. 3.1), can be explored toward the creation of efficient selective membranes for gas separation. Some well established and widely used membranes such as the polymeric ones, may exhibit higher selectivities compared to the ones presented in this study, but they can not be typically used in processes at high temperatures and pressures (operating conditions common in the industry), limiting their wider application [33-35]. The combination of properties exhibited by the PF-based superstructures certainly make them potential candidates for the next generation of materials for efficient and versatile gas separation membranes.

\section{Conclusions}

We have introduced a novel class of carbon-based nanoporous materials, comprising a variety of 1-D, 2-D and 3-D superstructures created upon covalent bonding of porous fullerenes, employing different types of covalent connections ( $s p^{2}$ and $s p^{3}$ hybridized) and geometric patterns. We show that the type of fullerene interconnection used and the structural dimensionality result in materials with varying properties. In general, they possess a well-defined pore size distribution, low density, high mechanical strength (associated with high flexibility in some cases), and electronic behavior ranging from semiconducting to metallic. This interesting combination of properties can be exploited toward the development of functional materials for many potential applications, including nanosensors, nanoelectronics, gas separation, gas storage/adsorption, support for catalysts, among others [1-9].

We hope that the presented results motivate future studies toward the realization of these materials in laboratory and development of practical applications, as well as theoretical studies focused on possible processes for controlled synthesis of these 
nanostructures. The success in the synthesis of $\mathrm{C}_{60}$ fullerene polymers using distinct approaches [17,18,21] certainly brings optimistic expectations on that front. Also, future theoretical studies can be carried out in order to evaluate the performance of these materials in specific applications, such as gas storage, catalyst supports and sensors, which will certainly brings extra motivation toward the development of efficient processes for production of these nanostructures.

\section{Acknowledgments}

RP acknowledges FAPESP for financial support through grant 2014/15521-9. This research was supported in part by resources supplied by the Center for Scientic Computing (NCC/GridUNESP) of the São Paulo State University (UNESP). ARM acknowledges grant 449824/2014-4 Chamada MCTI/CNPQ/UNIVERSAL 14/2014. The authors acknowledge computational support from CESUP/UFRGS/ Brazil and LNCC/SantosDumont.

\section{Appendix A. Supplementary data}

Supplementary data related to this article can be found at https://doi.org/10.1016/j.carbon.2018.01.041.

\section{References}

[1] G. Lu, X.S. Zhao, Nanoporous Materials: Science and Engineering, vol. 4, World Scientific, 2004.

[2] R.E. Morris, P.S. Wheatley, Gas storage in nanoporous materials, Angew. Chem. Int. Ed. 47 (27) (2008) 4966-4981.

[3] Y. Yang, K. Chiang, N. Burke, Porous carbon-supported catalysts for energy and environmental applications: a short review, Catal. Today 178 (1) (2011) 197-205.

[4] S.T. Meek, J.A. Greathouse, M.D. Allendorf, Metal-organic frameworks: a rapidly growing class of versatile nanoporous materials, Adv. Mater. 23 (2) (2011) 249-267.

[5] A. Ismail, L. David, A review on the latest development of carbon membranes for gas separation, J. Membr. Sci. 193 (1) (2001) 1-18.

[6] S.H. Joo, S.J. Choi, I. Oh, J. Kwak, et al., Ordered nanoporous arrays of carbon supporting high dispersions of platinum nanoparticles, Nature 412 (6843) (2001) 169.

[7] R. Lv, E. Cruz-Silva, M. Terrones, Building complex hybrid carbon architectures by covalent interconnections: graphene-nanotube hybrids and more, ACS Nano 8 (5) (2014) 4061-4069.

[8] L. Jiang, Z. Fan, Design of advanced porous graphene materials: from graphene nanomesh to 3d architectures, Nanoscale 6 (2014) 1922-1945.

[9] K. Goh, H.E. Karahan, L. Wei, T.-H. Bae, A.G. Fane, R. Wang, Y. Chen, Carbon nanomaterials for advancing separation membranes: a strategic perspective, Carbon 109 (2016) 694-710.

[10] M. Tagami, Y. Liang, H. Naito, Y. Kawazoe, M. Kotani, Negatively curved cubic carbon crystals with octahedral symmetry, Carbon 76 (2014) 266-274.

[11] D.C. Miller, M. Terrones, H. Terrones, Mechanical properties of hypothetical graphene foams: giant schwarzites, Carbon 96 (2016) 1191-1199.

[12] R. Paupitz, C.E. Junkermeier, A.C. van Duin, P.S. Branicio, Fullerenes generated from porous structures, Phys. Chem. Chem. Phys. 16 (46) (2014) $25515-25522$.

[13] M. Bieri, M. Treier, J. Cai, K. Ait-Mansour, P. Ruffieux, O. Groening, P. Groening, M. Kastler, R. Rieger, X. Feng, K. Muellen, R. Fasel, Porous graphenes: twodimensional polymer synthesis with atomic precision, Chem. Commun. 45 (2009) 6919-6921.

[14] G. Brunetto, P.A.S. Autreto, L.D. Machado, B.I. Santos, R.P.B. dos Santos, D.S. Galvao, Nonzero gap two-dimensional carbon allotrope from porous graphene, J. Phys. Chem. C 116 (23) (2012) 12810-12813.

[15] H.W. Kroto, J.R. Heath, S.C. Obrien, R.F. Curl, R.E. Smalley, C-60-buckminsterfullerene, Nature 318 (6042) (1985) 162-163.

[16] V.R. Coluci, R.P.B. dos Santos, D.S. Galvao, Topologically closed macromolecules made of single walled carbon nanotubes-'super'-fullerenes, J. Nanosci. Nanotechnol. 10 (7) (2010) 4378-4383.

[17] P.C. Eklund, A. Rao, Fullerene Polymers and Fullerene Polymer Composites vol. 38, Springer Science \& Business Media, 2013.

[18] F. Giacalone, N. Martn, Fullerene polymers: synthesis and properties, Chem. Rev. 106 (12) (2006) 5136-5190.

[19] V.R. Coluci, N.M. Pugno, S.O. Dantas, D.S. Galvao, A. Jorio, Atomistic simulations of the mechanical properties of 'super' carbon nanotubes, Nanotechnology 18 (33) (2007) 335702.

[20] Y. Li, X. Qiu, Y. Yin, F. Yang, Q. Fan, The elastic buckling of super-graphene and super-square carbon nanotube networks, Phys. Lett. 374 (15-16) (2010) $1773-1778$

[21] E. Hernndez, V. Meunier, B.W. Smith, R. Rurali, H. Terrones, M. Buongiorno Nardelli, M. Terrones, D.E. Luzzi, J.-C. Charlier, Fullerene coalescence in nanopeapods: a path to novel tubular carbon, Nano Lett. 3 (8) (2003) 1037-1042.

[22] S. Plimpton, Fast parallel algorithms for short-range molecular-dynamics, J. Comput. Phys. 117 (1) (1995) 1-19.

[23] S.J. Stuart, A.B. Tutein, J.A. Harrison, A reactive potential for hydrocarbons with intermolecular interactions, J. Chem. Phys. 112 (14) (2000) 6472-6486.

[24] A.C. Van Duin, S. Dasgupta, F. Lorant, W.A. Goddard, Reaxff: a reactive force field for hydrocarbons, J. Phys. Chem. 105 (41) (2001) 9396-9409.

[25] S. K. Singh, S. G. Srinivasan, M. Neek-Amal, S. Costamagna, A. C. T. van Duin, F. M. Peeters, Thermal properties of fluorinated graphene, Phys. Rev. B 87 (10).

[26] R. Smith, K. Jolley, C. Latham, M. Heggie, A. van Duin, D. van Duin, H. Wu, A reaxff carbon potential for radiation damage studies, Nucl. Instrum. Methods Phys. Res. Sect. B Beam Interact. Mater. Atoms 393 (2017) 49-53.

[27] A.R. Muniz, A.S. Machado, D. Maroudas, Mechanical behavior of interlayerbonded nanostructures obtained from bilayer graphene, Carbon 81 (2015) 663-677.

[28] J.F. Silveira, A.R. Muniz, First-principles calculation of the mechanical properties of diamond nanothreads, Carbon 113 (2017) 260-265.

[29] A. Cheng, W. Steele, Computer simulation of ammonia on graphite. i. low temperature structure of monolayer and bilayer films, J. Chem. Phys. 92 (6) (1990) 3858-3866.

[30] W.L. Jorgensen, D.S. Maxwell, J. Tirado-Rives, Development and testing of the opls all-atom force field on conformational energetics and properties of organic liquids, J. Am. Chem. Soc. 118 (45) (1996) 11225-11236.

[31] S. Jiao, Z. Xu, Selective gas diffusion in graphene oxides membranes: a molecular dynamics simulations study, ACS Appl. Mater. Interfaces 7 (17) (2015) 9052-9059.

[32] H.J. Berendsen, J. v. Postma, W.F. van Gunsteren, A. DiNola, J. Haak, Molecular dynamics with coupling to an external bath, J. Chem. Phys. 81 (8) (1984) 3684-3690.

[33] P. Bernardo, E. Drioli, G. Golemme, Membrane gas separation: a review/state of the art, Ind. Eng. Chem. Res. 48 (10) (2009) 4638-4663.

[34] D.F. Sanders, Z.P. Smith, R. Guo, L.M. Robeson, J.E. McGrath, D.R. Paul, B.D. Freeman, Energy-efficient polymeric gas separation membranes for a sustainable future: a review, Polymer 54 (18) (2013) 4729-4761.

[35] Y. Yampolskii, Polymeric gas separation membranes, Macromolecules 45 (8) (2012) 3298-3311.

[36] P. Giannozzi, S. Baroni, N. Bonini, M. Calandra, R. Car, C. Cavazzoni, D. Ceresoli, G.L. Chiarotti, M. Cococcioni, I. Dabo, et al., Quantum espresso: a modular and open-source software project for quantum simulations of materials, J. Phys. Condens. Matter 21 (39) (2009) 395502.

[37] S. Ryu, M.Y. Han, J. Maultzsch, T.F. Heinz, P. Kim, M.L. Steigerwald, L.E. Brus, Reversible basal plane hydrogenation of graphene, Nano Lett. 8 (12) (2008) 4597-4602.

[38] Y. Gogotsi, V. Presser, Carbon Nanomaterials, second ed., CRC Press, 2014

[39] B.D. Jensen, K.E. Wise, G.M. Odegard, Simulation of the elastic and ultimate tensile properties of diamond, graphene, carbon nanotubes, and amorphous carbon using a revised reaxff parametrization, J. Phys. Chem. 119 (37) (2015) 9710-9721.

[40] B. WenXing, Z. ChangChun, C. WanZhao, Simulation of young's modulus of single-walled carbon nanotubes by molecular dynamics, Phys. B Condens. Matter 352 (1) (2004) 156-163.

[41] D.-e. Jiang, V.R. Cooper, S. Dai, Porous graphene as the ultimate membrane for gas separation, Nano Lett. 9 (12) (2009) 4019-4024.

[42] D. Cohen-Tanugi, J.C. Grossman, Water desalination across nanoporous graphene, Nano Lett. 12 (7) (2012) 3602-3608.

[43] L. Huang, M. Zhang, C. Li, G. Shi, Graphene-based membranes for molecular separation, J. Phys. Chem. Lett. 6 (14) (2015) 2806-2815. 